\title{
Calorimetry at the ILC detectors
}

\author{
Sergej Schuwalow* \\ DESY, Platanenallee 6, 15738, Zeuthen, Germany
}

\section{A R T I C L E I N F O}

Available online 27 August 2008

\section{Keywords:}

ILC

Calorimetry

PFA

\begin{abstract}
A B S T R A C T
The current status of the research work on the ILC Calorimetry is described. Several sub-detectors are addressed: ECAL, HCAL, LumiCal, BeamCal and GamCal. As ECAL and HCAL are concerned, two major concepts are discussed: Particle Flow Approach and Multiple Readout calorimetry. Results from beam tests are reported.
\end{abstract}

(c) 2008 Elsevier B.V. All rights reserved.

\section{Introduction}

Calorimeters of the ILC detectors serve for a precise jet energy measurement, for the accurate and fast measurement of the luminosity, and to ensure hermeticity down to small polar angles. To fully exploit the physics potential of the ILC, the resolution of the jet energy measurement, $\sigma_{E} / E$, is required to be $\approx 30 \% / \sqrt{(E)}$ or $3-4 \%$ at energies above $100 \mathrm{GeV}$. This resolution, being about a factor of two smaller as the best currently operating calorimeters, must be maintained almost over the full polar angle range. The ability to detect single high energy electrons with nearly $100 \%$ efficiency is required at very small polar angles to ensure the potential for new particle searches. Special calorimeters in the very forward region are foreseen for these purposes. They will also perform fast and precise measurement of the delivered luminosity.

To approach the required jet energy resolution, research is done for two different calorimeter concepts. The first, followed by the majority of R\&D projects, is the development of extremely fine grained and dense calorimeters with single particle shower imaging. The particle flow approach (PFA) is used to determine the jet energy and direction. Tracks are matched to their depositions inside the calorimeters. Showers without matched tracks are assumed to originate from neutral particles. The jet energy is then composed from the best measured observables of the jet components: momenta of charged particles as measured by the tracker and the depositions from neutral particles in the calorimeters.

Separation of showers requires the smallest possible Moliere radius, a relatively large distance between IP and the calorimeter and a strong magnetic field. PFA is shown to be very sensitive to the material budget before the calorimeter, so in all existing detector designs calorimeters are placed inside the magnet coil

\footnotetext{
* Tel.: +49 3376277396 ; fax: +49337627 7330 .

E-mail address: sschuwal@ifh.de
}

and special care is taken to make the tracker as thin as possible. Using Monte Carlo simulations it has been demonstrated that a significant improvement of the jet energy resolution is feasible, but substantially more effort is needed to optimize the calorimeter design and to improve the particle flow algorithms. In addition Monte Carlo simulations must be verified by test-beam measurements.

The second concept, followed by one group, exploits the dual readout of scintillation and Cherenkov light of fibers or crystals (DREAM). The electromagnetic and hadronic component inside a shower can be independently measured and finally properly recombined with a gain in resolution due to reduced fluctuations.

In many cases the R\&D work has not yet reached into a point where a final choice of technologies can be made, so several alternative solutions are presented.

\section{External constraints}

The design of the ILC accelerator based on the superconducting cavities leads to a specific bunch structure and a repetition rate which is favorable for the calorimeter readout. The train repetition rate is $5 \mathrm{~Hz}$ with 2820 bunches per train. The interval between bunch crossings is $308 \mathrm{~ns}$, each train has $0.87 \mathrm{~ms}$ duration followed by a $199 \mathrm{~ms}$ collision-free gap. A common feature for all readout designs is fast $\mathrm{FE}$ electronics which is able to store complete train information and a separate readout chain to transmit data to DAQ system during inter-train gaps. Large idle time of about $99.5 \%$ allows a power save mode, when significant part of readout electronics is powered only during bunch trains.

The detector design with a solenoid outside the calorimeter suggests an overall cylindrical symmetry, which for technological reasons is often approximated by octagonal or dodecagonal shape. Usually the calorimeter is subdivided into a cylindrical barrel and two end-caps. 


\section{Electromagnetic calorimeters for particle flow approach}

Electromagnetic calorimeters (ECAL) are designed as compact and fine-grained sandwich calorimeters optimized for the reconstruction of photons and electrons. Since about $60 \%$ of hadrons will interact in the ECAL, good shower imaging capability is also required. To keep the Moliere radius near the minimum possible tungsten or lead are used as absorber. Sensor planes are made of silicon pad diodes, monolithic active pixel sensors (MAPS) or of scintillator strips or tiles. Also the combination of silicon and scintillator sensor planes was investigated. The range of energies of electrons and photons suggests a thickness of about 24 radiation length for the ECAL. To reach adequate energy and position resolution over the necessary energy range, the sampling thickness should be fine enough at least for the front part of ECAL.

\subsection{Silicon tungsten sandwich calorimeter}

Two groups study silicon tungsten calorimeters in detail. The first, within the CALICE [1] collaboration, proposes a mechanical frame made of carbon fiber reinforced epoxy with integrated tungsten absorber plates. Between the absorber plates space is left for detector slabs, containing silicon sensor planes. The silicon sensors are structured with quadratic pads of $5 \times 5 \mathrm{~mm}^{2}$ size, being about a third of the ECAL Moliere radius, and about $300 \mu \mathrm{m}$ thickness. The sensors are glued to a PCB which carries the frontend electronics ASICs. Data are processed in the front-end ASIC and concentrated by a chip on the edge of the detector slab. The concentrator flushes the data after the bunch train and sends them to the DAQ (Figs. 1 and 2).

CALICE has built a prototype calorimeter with sensors of $1 \times 1 \mathrm{~cm}^{2}$ pad size and took data in a $5 \mathrm{GeV}$ electron beam at DESY and in a higher energy electron and hadron test-beams at CERN together with an AHCAL prototype module. More than $200 \mathrm{M}$ events are under analysis. Preliminary results of $17 \% / \sqrt{E} \oplus 1 \%$ energy resolution and good linearity had been reported. Further tests of ECAL and electronics are planned at Fermilab test beam in 2008.

CALICE prepares in parallel a second prototype, called EUDET module, which will be as close as possible to the final design. This is a full length structure, partly equipped with sensors and absorbers. The pad size is $5 \times 5 \mathrm{~mm}^{2}$, resulting in $40 \mathrm{k}$ channels to be readout. The construction and test of the EUDET module will be the final proof of the CALICE ECAL design.

The second project is pursued by groups collaborating on the Silicon Detector (SiD) Design Study [2]. The mechanical structure is built of tungsten planes, fixed to each other by rods. A $1 \mathrm{~mm}$ gap is left for the silicon sensor planes. The total thickness of the

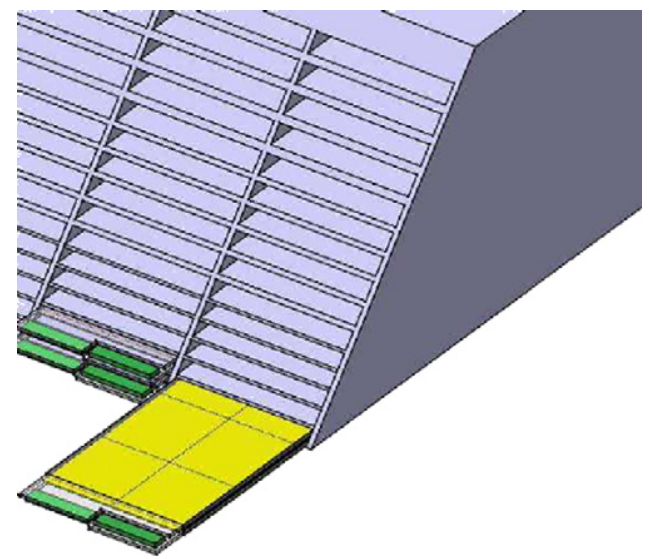

Fig. 1. The structure of the silicon tungsten calorimeter, as proposed by the CALICE collaboration. The slots in the tungsten frame are equipped with detector slabs.

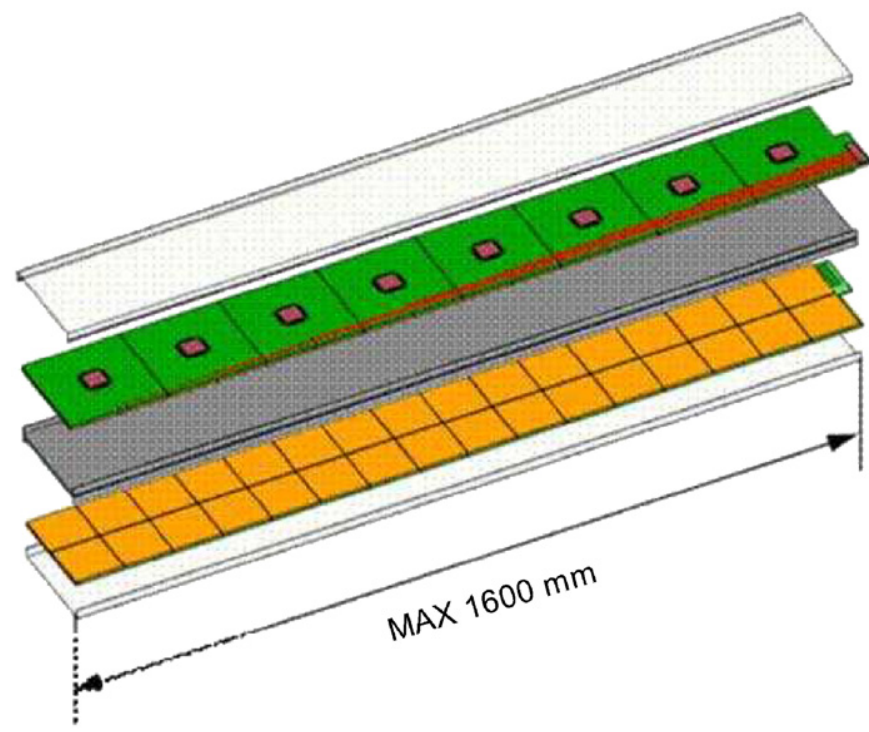

Fig. 2. A single detector slab which will be inserted into the ECAL support structure. The tungsten absorber plate (gray) is attached at both sides by silicon pad sensors with FE chips on top.

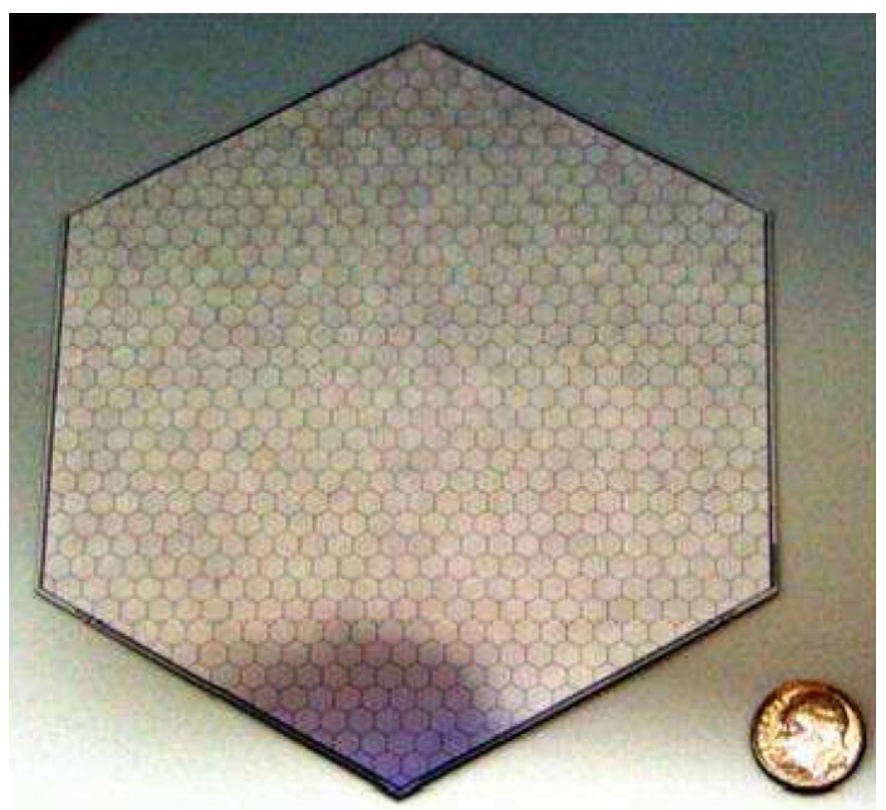

Fig. 3. A silicon sensor prototype with hexagonal pads for Silicon Detector ECAL. 1024-channel KPiX R/O chip will be placed at the center.

tungsten absorber corresponds to $27 X_{0}$. The expected energy resolution is $15 \% / \sqrt{E} \oplus 1 \%$.

The sensor layer consists of hexagonal silicon planes made from 6 in wafers, maximizing the use of a round wafer, as shown in Fig. 3. The silicon plane is subdivided in 1024 hexagonal diodes of $13 \mathrm{~mm}^{2}$ area each. Each plane will be readout by one 1024 channel ASIC (KPiX) positioned at the center. The KPiX chip performs the analog condition ing and 15-bit digitization for all channels and provides time stamping of bunch crossings. The KPiX chip has a feature of the switching gain dynamically, which accommodates the large dynamic range required for the ECAL. The data are serialized and transported by transverse data cables to the edge of a calorimeter module.

It is worth to note that KPiX has been adapted for use with silicon microstrip detectors and RPC and GEM detectors for the hadronic calorimeter and muon system. The KPiX-6 version of the 
chip with $2 \times 32$ channels is under test, full scale prototype submission will be done in 2008 .

The goal of the R\&D is to fabricate a full-depth electromagnetic calorimeter prototype module. This will consist of 30 longitudinal layers, each consisting of an about $15 \mathrm{~cm}$ diameter silicon detector equipped with a KPiX chip sandwiched between $2.5 \mathrm{~mm}$ thick tungsten absorber layers. The module will be fully characterized for electromagnetic response and resolution in an electron beam in 2009. A first round of 10 silicon detectors, made from a 6 in wafer, has been tested in the laboratory, and a second round of 40 sensors made by Hamamatsu is expected in 2008.

\subsection{Monolithic active pixel digital ECAL}

A group from RAL within the CALICE Collaboration has proposed to use monolithic active pixel sensors (MAPS) instead of silicon pad diodes for the ECAL. MAPS are produced in CMOS technology which is widely used in semiconductor industry and combine the detector and readout in the same chip. To instrument an ECAL with MAPS might be of lower cost than using the high resistivity silicon needed for the previous designs. The readout of the pixel will be binary. To ensure that a pixel inside a shower is mostly hit only by one particle, the pixel size must be about $50 \times 50 \mu \mathrm{m}^{2}$. The total number of pixels for the ECAL will be about $8 \times 10^{11}$. The hits during a bunch train are stored on the sensor with time stamps and hit pixel numbers and readout between trains. The expected DAQ data rate is $\sim 400 \mathrm{Gbit} / \mathrm{s}$ mainly due to noise and beam background.

The first $168 \times 168$ pixel TPAC1 version of the chip has been investigated in the laboratory using radioactive sources, cosmics and laser beams. Test beam measurements are planned at DESY in 2008. The power consumption of the TPAC1 chip is about $40 \mu \mathrm{W} / \mathrm{mm}^{2}$ and expected to be significantly lower for the next version.

The digital approach may have big advantages e.g. to facilitate the calibration of the ECAL and to ensure excellent shower image reconstruction.

\subsection{Scintillator tungsten sandwich calorimeter}

For a calorimeter with a large radius, a finely segmented scintillator-based sandwich calorimeter may have a particle flow performance similar to a compact silicon-tungsten calorimeter, but might have better resolution and lower cost. A group of Asian Labs within CALICE [3,4] plans a sandwich calorimeter using plastic scintillator as a sensor.

Layers of scintillator strips, oriented perpendicular to each other as shown in Fig. 4, are placed in between tungsten absorber plates. The effective segmentation given by the strip width is $1 \times 1 \mathrm{~cm}^{2}$. Each strip is equipped with a wavelength-shifting (WLS) fiber readout by novel Geiger mode photo-diodes, MPPC.

A prototype calorimeter read-out with classical multi-anode photo-multipliers was tested in an electron beam of energies from 1 to $4 \mathrm{GeV}$ at KEK. The energy and shower position resolutions of about $13 \% / \sqrt{E}$ and $4.5 / \sqrt{E} \mathrm{~mm}$, respectively, agreed perfectly with Monte Carlo simulations. In addition, the angle of the shower axis was measured with a resolution of $4.8 / \sqrt{E}$ degrees, demonstrating the ability to detect photons not originating from the interaction point [5].

A new prototype calorimeter has been instrumented with MPPCs and tested in the beam at DESY in 2007. The thickness of the tungsten absorber plates was $3.5 \mathrm{~mm}$ and the thickness of the scintillator strips was $3 \mathrm{~mm}$. Part of scintillator strips were made from large planes structured by grooves with WLSF or direct light readout, the rest of strips were made separate using an extrusion technology and readout by WLS fibers. MPPC sensors with 1600

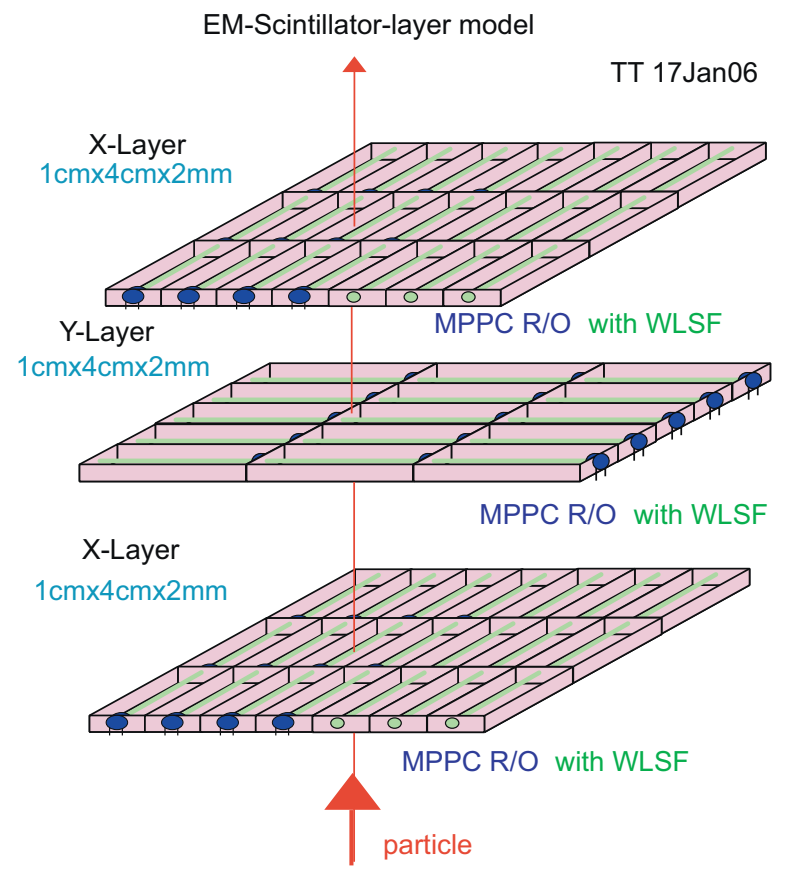

Fig. 4. A possible scintillator strip sequence of the ECAL for GLD. Each strip is equipped with a WLS fiber (green) and readout by a MPPC.

pixels were delivered by Hamamatsu. Experience obtained in this test-beam study will be used for the construction of a larger prototype to be tested at FNAL in 2008 together with CALICE AHCAL.

A group from the University of Colorado [6] proposed a scintillator-tungsten sandwich calorimeter using scintillator tiles of $5 \times 5 \mathrm{~cm}^{2}$ size readout with silicon photo-detectors, SiPDs, via WLS fiber. In order to improve the shower position resolution the tiles in consecutive layers are displaced by $2.5 \mathrm{~cm}$. A small tile module was operated in the lab and performance studies are done with cosmic rays.

\subsection{Mixed silicon and scintillator lead ECAL}

A prototype of a sandwich calorimeter consisting of 45 scintillator planes and three planes of silicon pads interspersed between lead absorber plates was built and operated in a testbeam [7]. Scintillator planes were subdivided into $5 \times 5 \times 0.3 \mathrm{~cm}^{3}$ cells with WLS fiber readout. Three planes of silicon diode pads $\left(0.9 \times 0.9 \mathrm{~cm}^{2}\right)$ were inserted at a depth of 2,6 and $12 X_{0}$. The measured energy resolution was parametrized as $11 \% / \sqrt{E}$. There is no plan for the moment to continue the project.

\section{Hadron calorimeter for particle flow approach}

Several technologies of fine-segmented sampling calorimeters are under investigation with either analog or digital readout. The analog read out calorimeters use scintillator tiles or scintillator strips as sensors. Digital calorimeters use GEMs (Gaseous Electron Multipliers), Micromegas (Micro mesh gaseous structures) or RPCs (Resistive Plate Chambers) as active elements.

\subsection{Analog $H C A L$}

Analog hadron calorimeters use scintillator as detector and steel or lead as absorber. The scintillator tiles are readout by novel photo-sensors, e.g. MPPCs, SiPDs or Silicon Photo-multipliers (SiPMs). These photo-sensors are similar but developed by different producers. 
Two projects are pursued within CALICE. One is based on small area scintillator tiles of a several mm thickness read out by SiPMs via WLS fibers. Layers of stainless steel serve as absorber and form the mechanical frame.

Currently a $1 \mathrm{~m}^{3}$ prototype calorimeter has been equipped with $1 \mathrm{~m}^{2}$ sensor layers and tested in CERN lepton and hadron beams. The scintillator tiles are of $3 \times 3 \mathrm{~cm}^{2}$ size in the core of the calorimeter and $10 \times 10 \mathrm{~cm}^{2}$ in the edge regions. The granularity in the core has been chosen to optimize the particle shower separation power. It is also small enough to test semi-digital (2-bit) readout concepts. Each tile is equipped with a SiPM. The signals are transported using thin wires to one side of the plane and feed in the DAQ electronics which is the same as the one being used for the CALICE silicon-tungsten ECAL prototype. The calorimeter is supplemented by a tail catcher and muon tracker to ensure full measurement of hadron showers.

About 200 million events with electron and hadron beams in the energy range from 6 to $80 \mathrm{GeV}$ have been collected at CERN in 2006/2007, mostly in conjunction with the silicon-tungsten ECAL. These data already allow first studies of particle flow performance with ECAL and HCAL together. The prototype instrumentation was completed in 2007, further beam tests together with different ECAL prototypes are planned in 2008/2009 at Fermilab.

A versatile mechanical support structure is under construction, which will make studies with inclined beam incidence possible. The same structure shall later also be used with gaseous HCAL modules, described below, for a direct comparison with the purely digital options. Further steps will include the construction of full scale Technical Prototype which is shown in Fig. 5. A half-barrel size mechanical structure will be partly equipped with readout planes with embedded electronics and beam-tested in 2009/2010.

The second project is focused on a hadron calorimeter design which uses lead as absorber and scintillator layers as sensors. Lead is chosen to achieve hardware compensation, i.e. to ensure almost equal response for the electromagnetic and the hadronic shower component to reduce the fluctuations. Test-beam measurements have shown that compensation is achieved by choosing the ratio of lead-to-scintillator thickness to 9.1:2. In addition, plastic scintillator detects neutrons effectively, improving the energy measurement of hadrons. The scintillators are structured in strips and tiles as shown in Fig. 6. The strip width and length is set to 1 and $20 \mathrm{~cm}$, respectively, and are subject to ongoing optimization. Wavelength shifting fibers are placed inside grooves along the strip center or curled inside the tiles. To ensure a sufficient amount of light the scintillator thickness is set to $5 \mathrm{~mm}$.

The frontend electronics for test-beam studies of this HCAL design will be based on that developed for the CALICE ECAL.

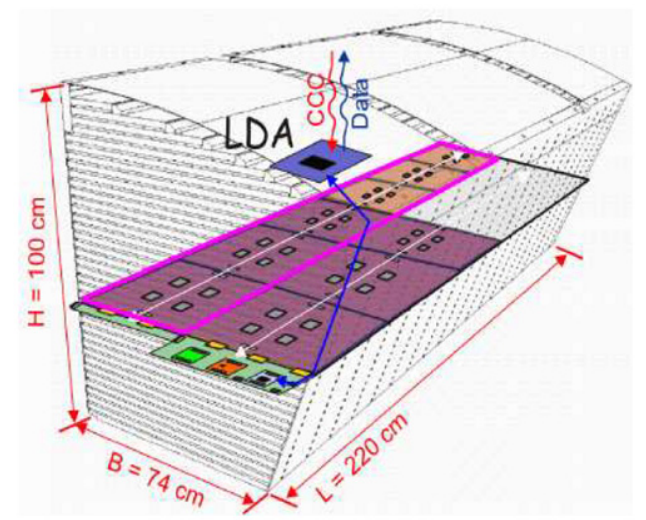

Fig. 5. Technical prototype of CALICE AHCAL module. Half-barrel size mechanical structure will be partly equipped with readout planes.

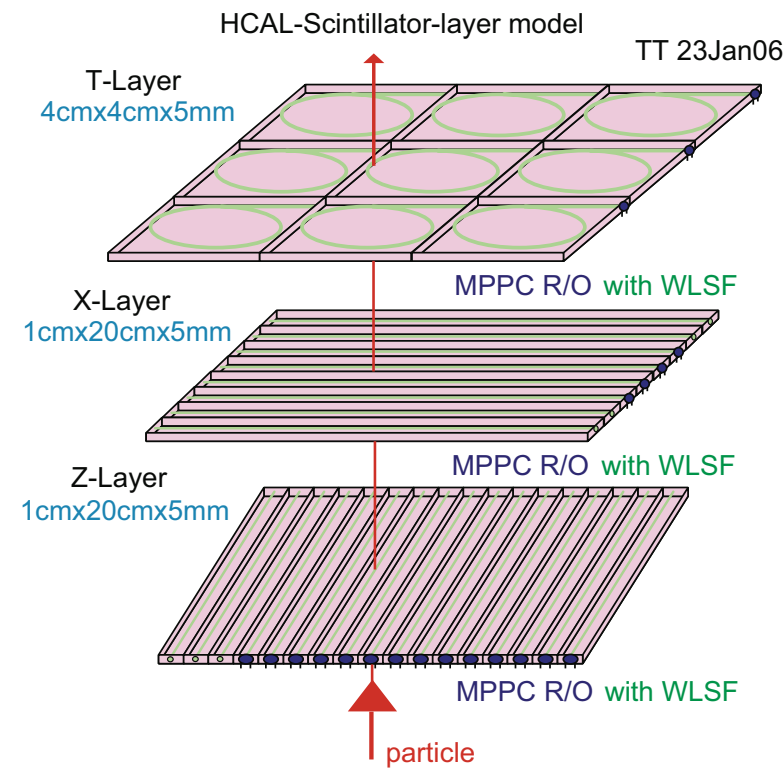

Fig. 6. A possible scintillator strip/tile sequence of the analog HCAL.

Test-beam data taken with a prototype calorimeter (probably in 2009/2010) will allow a detailed comparison to the steel/ scintillator calorimeter.

\subsection{Digital HCAL}

Going from analog readout to one-bit or two-bit readout electronics one can increase detector granularity and hence PFA performance while reducing cost. Energy resolution studies showed that pure digital 1-bit solution provides better resolution at low energy while analog option is favoured at high energy. The study of KEK group for the GLD HCAL using 2-bit, 3 thresholds $(.5,10,100 \mathrm{MIPs})$ associated to $1 \mathrm{~cm}^{2}$ tile size showed similar energy resolution with respect to the analog readout version for single particle and even better energy resolution for jets. In addition, a digital HCAL could be relatively easy calibrated in contrast to a very complicated and time consuming sandwich analog HCAL calibration [8].

Digital HCAL designs use gaseous signal amplification in GEMs, Micromegas or RPCs. Thin and large area chambers are placed between steel absorber plates. The chamber electrode is segmented in small pads of about $1 \mathrm{~cm}^{2}$ size, matching the granularity needed for the PFA application. Research work is done within the CALICE collaboration.

Signal amplification in GEM sensors occurs inside the holes in perforated Kapton foil metalized on both sides. Electrons created by ionization drift between the chamber cathode and the foil, induce an avalanche in the holes and then collected at the anode which has a pad structure. Several layers of GEM foils are used to achieve sufficient signal size. The size of the anode pads might be sufficiently small. GEMs provide fast signals and small recovery time. The pad signals are amplified and discriminated. If the signal of the pad is above the threshold it is counted as a hit.

Test chambers of area $30 \times 30 \mathrm{~cm}^{2}$, as shown in Fig. 7, are operated with cosmics, a radioactive source and a high intensity electron beam. A gas mixture of $80 \% \mathrm{Ar} / 20 \% \mathrm{CO}_{2}$ has been shown to work well with a gain of $10^{4}$, an efficiency of about $95 \%$ and a hit multiplicity of 1.27 . Chambers have been exposed to a high intensity electron beam. Even after collecting $2 \times 10^{12}$ electrons/pad, no decrease in gain was observed. 


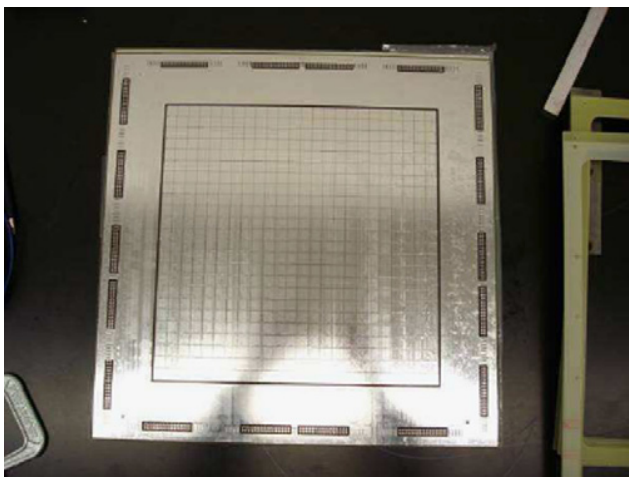

Fig. 7. A $30 \times 30 \mathrm{~cm}^{2}$ prototype of GEM chamber prepared for the test measurements.

Beam test of a $30 \times 30 \mathrm{~cm}^{2}$ GEM chamber at Fermilab is planned in 2008 using KPiX and DCAL ${ }^{1}$ chips for the readout. A $1 \mathrm{~m}^{3}$-size test beam module, planned for completion in 2009-2010, will be equipped with $100 \times 33 \mathrm{~cm}^{2}$ chambers and beam-tested as a part of CALICE stack. Major activities are ongoing for the mechanical aspects of a large GEM-layer assembly and the fabrication of large area GEM foils in collaboration with an industrial company in the US.

In Micromegas electrons produced by ionizing particle drift in the gas volume between the cathode and micromesh layer. After crossing micromesh they get into the mesh-anode gap with high electric field and induce an avalanche. Signals are picked up from anode strips or pads. The gap between the mesh and the anode can be made in the range of $100 \mu \mathrm{m}$, leading to a small size avalanche and excellent spatial resolution and potentially low pad multiplicity. Chambers based on Micromegas can be made very thin, about $4 \mathrm{~mm}$, allowing to built a very compact calorimeter. Test chambers as shown in Fig. 8 are of $50 \times 50 \mathrm{~cm}^{2}$ size. They are being prepared for test-beam measurements in 2008. Provided the results from these measurements are satisfactory, a $1 \mathrm{~m}^{2}$ plane will be built for test-beam studies in 2009/2010 as a part of DHCAL CALICE module.

In RPCs a homogeneous electric field is created in the wide gap between precise glass plates covered by resistive paint. The gap is filled with a working gas mixture. Ionizing particle crossing the gap induce a local discharge, an image charge of it is picked up by the pad structure outside the chamber. Due to homogeneous electric field RPCs require much higher voltage to be applied with respect to GEM and Micromegas options.

Prototypes of different size RPCs are shown in Fig. 9.

Tests have been carried out with cosmic rays, sources, and a particle beam. The efficiency, measured in a proton beam of $120 \mathrm{GeV}$ at FNAL, approaches nearly $100 \%$ above $7 \mathrm{kV}$ of applied voltage. The pad multiplicity for a $1 \times 1 \mathrm{~cm}^{2}$ pads ranges between 1.1 and 1.6, depending on the high voltage and on the particular design of the chamber. The stack of nine fully equipped chambers interleaved with $20 \mathrm{~mm}$ steel-copper absorber plates has been beam-tested at FNAL in summer 2007 (Vertical Slice test). Chambers were readout by the DCAL chip. A $1 \mathrm{~m}^{3}$ RPC DHCAL prototype with $\sim 400 \mathrm{k}$ channels is planned to be constructed in 2008-2009 and beam-tested as a part of the CALICE stack in 2009.

\section{DREAM calorimeter}

The DREAM collaboration [9] follows a fundamentally different concept to improve the jet energy resolution. Usual hadron

\footnotetext{
${ }^{1}$ 64-channel DCAL chip is developed by ANL/FNAL for digital ILC calorimetry.
}

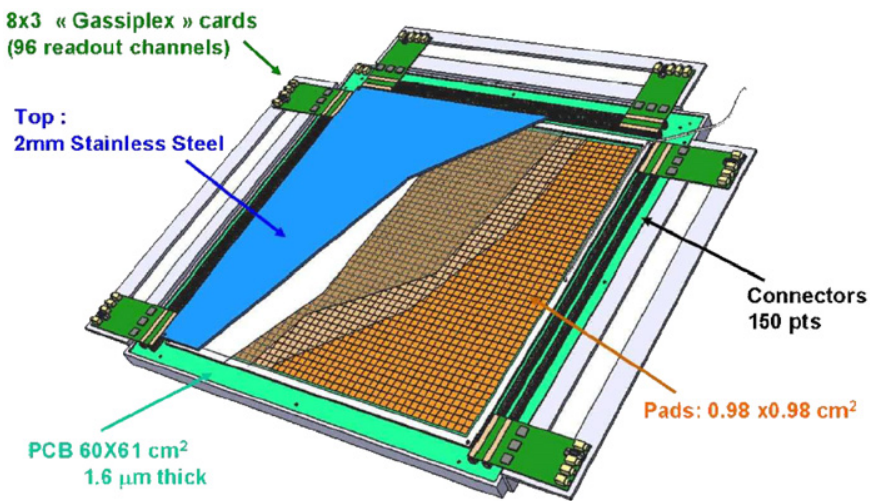

Fig. 8. A $50 \times 50 \mathrm{~cm}^{2}$ Micromegas chamber. FE readout electronics is placed at the chamber frame.

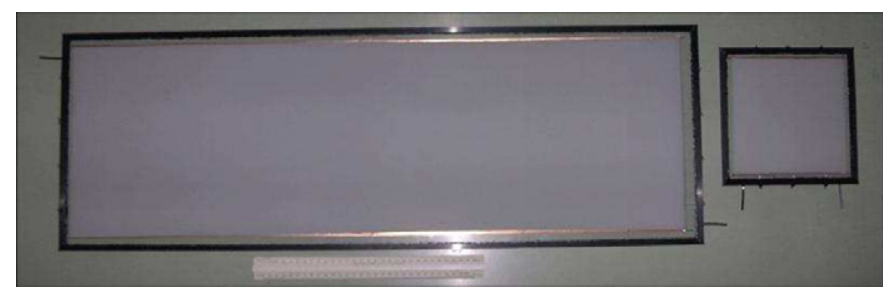

Fig. 9. Prototypes of RPCs for performance studies. The larger chamber size is $100 \times 30 \mathrm{~cm}^{2}$.

sampling calorimeters are limited in the energy resolution due to fluctuations induced by the different response from the electromagnetic and hadronic shower component. DREAM uses a dual readout concept. The sensors inside the absorber are scintillation and clear fibers readout separately. Scintillating fibers respond to all charged particles in a shower while clear fibers detect Cherenkov light induced mainly by electrons and positrons. Because of the fine-grained spatial sampling also fluctuations in the local energy depositions are accounted for. In addition, the detection of MeV neutrons in e.g. hydrogen enriched fibers, might further improve the energy resolution.

Besides the excellent energy resolution, the dual-readout calorimeter has very good linearity and gaussian response for given energy, which may easy the calibration with respect to sandwich calorimeters [8].

The DREAM Collaboration performs test-beam studies both with scintillating and clear fibers inside a copper absorber. The bundles of clear and scintillating fibers are read out by photomultipliers. Measurements were done with muons, electrons and hadrons. As an example, the measured resolution for hadrons as a function of the hadron energy using only the Cherenkov light follows a $86 \% / \sqrt{E} \oplus 10 \%$ dependence. Using the scintillator readout only the resolution can be parametrized as $49 \% / \sqrt{E} \oplus 7 \%$. Combining both in a proper way using the measured ratio of Cherenkov and scintillator light the resolution can be improved to $41 \% / \sqrt{E} \oplus 4.2 \%$. Further improvement of the latter result seems possible by reducing shower leakage, using e.g. a larger prototype, and by measuring neutron depositions.

For the ECAL section $\mathrm{PbWO}_{4}$ crystals are under study, read out with several photo sensors on their front and rear sides. Filters could enhance sensitivity to either scintillation light or to Cherenkov light exploiting the difference in their spectra. The different timing and the directionality of the Cherenkov light could also be exploited to improve the shower energy measurement in crystals.

DREAM promises an alternative to the particle flow concept. Studies are underway to characterize the performance of a 
calorimeter based on the DREAM technology in the ILC environment, e.g. in the reconstruction of multi-jet final states.

\section{Very forward calorimeters}

In the very forward region two calorimeters, BeamCal and LumiCal, are planned for a fast and precise measurement of the luminosity and ensure detector hermeticity [12]. A third calorimeter, GamCal, was proposed to support the fast luminosity measurement and beam parameter optimization. The first two calorimeters will be sampling calorimeters consisting of tungsten absorber disks interspersed with pad-structured solid-state sensor planes [13]. GamCal which is planned to be positioned $\sim 170 \mathrm{~m}$ downstream from the interaction point is still under design.

The BeamCal adjacent to the beam-pipe covers a polar angle down to about $5 \mathrm{mrad}$. Electron/positron pairs originating from beamstrahlung photon conversions deposit several $\mathrm{TeV}$ per bunchcrossing in the BeamCal. The distribution of this energy deposition will be measured to assist in tuning the beams. The expected dose collected is up to $10 \mathrm{MGy}$ per year for nominal accelerator parameters at $500 \mathrm{GeV}$ center-of-mass energy. Fine granularity and small Moliere radius is necessary to identify the localized depositions from high-energy two-photon event electrons [14] on top of the broader spread of energy depositions from relatively soft beamstrahlung pairs. The requirements on the sensors are stable operation under high electromagnetic doses, good linearity over a dynamic range of about $10^{4}$, very good homogeneity, and fast response. BeamCal has to be at least partly readout after each bunch-crossing requiring a specialized fast FE electronics [15]. Testbeam studies have been done using samples of CVD diamond sensors of $1 \mathrm{~cm}^{2}$ area and a few $100 \mu \mathrm{m}$ thickness. A reasonable linearity has been measured over a dynamic range of larger than $10^{5}$. The performance of several sensors as a function of the absorbed dose has been measured in a $10 \mathrm{MeV}$ electron beam for doses up to 7 MGy. For the sensors produced so far we observed a drop of the signal to about $30 \%$ and low noise [16]. Studies in close collaboration with the manufacturers are underway to improve the performance. In addition, alternatives like GaAs or special silicon are investigated. An example of a GaAs sensor designed for BeamCal is shown in Fig. 10. Several such sensors were studied at the test-beam in 2007.

The LumiCal is the luminometer of the detector and covers larger polar angle outside the reach of beamstrahlung pairs. The goal is to measure the luminosity by counting Bhabha events with an accuracy about $10^{-4}$. A silicon tungsten calorimeter has been simulated and e.g. requirements on the tolerances of the mechanical frame, the sensor positioning and the position of the calorimeters relative to the beam have been estimated. In particular the inner acceptance radius must be controlled at the $\mu \mathrm{m}$ level.

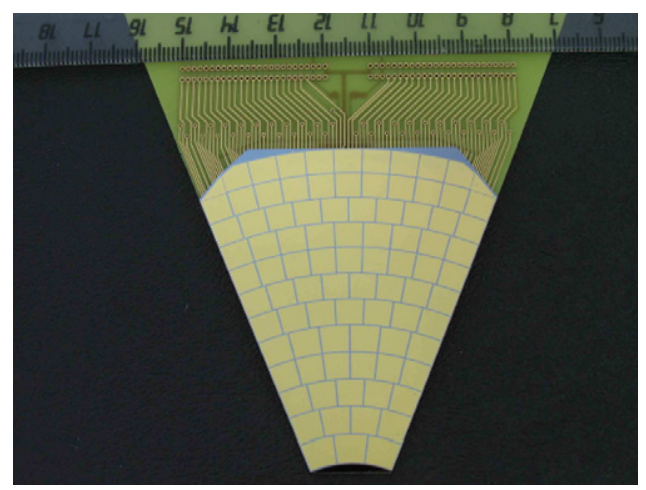

Fig. 10. Prototype of GaAs sensor tile designed for BeamCal.

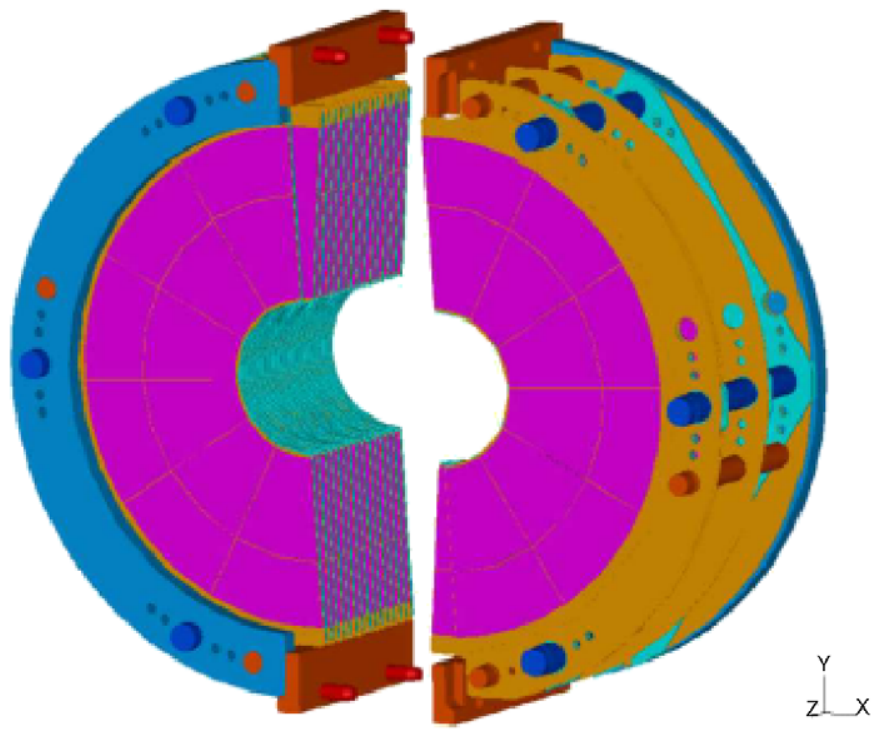

Fig. 11. Mechanical structure of LumiCal. Sensor planes have to be positioned with $\mu \mathrm{m}$ accuracy.

The mechanical design is shown in Fig. 11. The sensor layers will consist of pad-structured silicon sectors with extremely fine radial granularity. Prototypes of sensor planes will be available in 2008 for test measurements. First prototypes of FE electronics are currently under test.

\section{Conclusions}

The requirements on the performance of the calorimeters are physics driven. Potentially, all technologies pursued in the different collaborations and projects may match these requirements. To rank the proposed technologies further test-beam studies and full-system tests are necessary.

There is a large variety in the development level of the projects. CALICE took data with the first prototype for an ECAL and an analog HCAL in a test-beam and will be able to answer many questions using these data in the near term. Other projects are going to built prototypes in the near future and test them in beams.

Tests of the DREAM concept continue. To demonstrate the feasibility of this technology for the ILC, a design suitable for a collider detector should be worked out which provides the performance demanded by ILC physics.

The special calorimeters to instrument the very forward region are still in a development phase. Ongoing sensor tests are necessary to make a suitable choice. Construction of prototypes for beam tests will be the next important future step.

\section{References}

[1] CALICE 〈http://llr.in2p3.fr/activites/physique/flc/calice.html>.

[2] SiD 〈http://silicondetector.org/display/SiD/Calorimeter).

[3] GLD 〈http://ppwww.phys.sci.kobe-u.ac.jp/ kawagoe/gldcal/>.

[4] The KEK Detector Technology Project 〈http://rd.kek.jp〉.

[5] A. Nagano, et al., Nucl. Instr. and Meth. A 557 (2006) 460.

[6] 〈http://www-hep.colorado.edu/experimental/ilc/ 〉.

[7] M. Alemi, et al., LC-DET-2004026 (2004).

[8] R. Wigmans, AIP Conf. Proc. 867 (2006) 90

[9] N. Akchurin, et al., Nucl. Instr. and Meth. A 584 (2008) 304.

[12] H. Abramovicz, et al., IEEE Trans Nucl. Sci. NS-51 (2004) 2983.

[13] FCAL Collaboration 〈http://www-zeuthen.desy.de/ILC/fcal/>.

[14] W.Lohmann: DESY-05-142.

[15] C. Grah, A. Sapronov: EUROTeV-Report-2007-006.

[16] C. Grah, et al., IEEE NSS07, November 2007, Submitted for publication. 